\title{
Survey of Real-Time Communication in CSMA-Based Networks
}

\author{
Ricardo Moraes \\ Departamento de Ciência e Tecnologia, Universidade Federal de São Paulo \\ Rua Talim 330, 12231-280, São José dos Campos, São Paulo (Brazil) \\ Tel: 55-12-3942-5568Ｅ-mail: rmoraes@unifesp.br \\ Francisco Vasques and Paulo Portugal \\ Faculdade de Engenharia, Universidade do Porto \\ Rua Dr. Roberto Frias s/n, 4200-465, Porto (Portugal) \\ Tel: 351-22-508-1400Ｅ-mail: \{vasques, pportugal\}@fe.up.pt
}

\begin{abstract}
The purpose of this paper is to survey the state-of-the-art on real-time (RT) communication in CSMA-based networks and to identify the most suitable approaches to deal with the requirements imposed by next generation communication environments. The paper focuses in the two of the most relevant solutions that operate in shared broadcast environments, according to the CSMA medium access protocol (IEEE 802.3/802.11). RT communication solutions are classified according to two classification axes. The first axis is related to how collisions are dealt with. A second classification axis is related to the compatibility degree achieved by each of the proposed approaches. Finally, a RT communication framework that is adequate to support RT communication in shared environments is briefly discussed.
\end{abstract}

Keywords: Real-Time communication, Ethernet networks, IEEE 802.3, IEEE 802.11. 


\section{Introduction}

During the last few years, the demand for real-time (RT) communication has been steadily increasing due to a new wide range of applications. Remarkable examples are VoIP (Voice over IP) and Networked Control Systems (NCS). For such type of applications, the support of timely communication services is one of the major requirements. For instance, in NCS applications, RT control data must be periodically transferred between sensors, controllers and actuators according to strict transfer deadlines. RT communication services are commonly classified according to the degree of RT guarantees into hard and soft RT groups. Hard RT applications require predictable and bounded response times, and violations of these response times may have severe consequences. Instead, soft RT applications can tolerate some losses of temporal deadlines. For instance, a RT control application can tolerate occasional losses of the control law updates, especially if the control law has been modified to account for those lost updates [1]. However, this type of applications is usually less resilient against jitter on the control law updates. In the case of a NCS, it is of utmost importance to have a nearly constant average communication delay and low jitter, whatever the behavior of the communication environment.

This paper surveys the state-of-the-art on RT communication in CSMA-based networks. From this survey, it becomes clear that traditional RT communication approaches for CSMA-based networks are not able to deal with the requirements imposed by next generation communication environments. More specifically, they are not able to handle uncontrolled traffic sources sharing the same broadcast environment. Similar conclusions could be drawn for other CSMA-based protocols, such as IEEE 802.15.4 (one of the most widespread PAN protocols) or CAN (commonly used in automotive and industrial applications). However, such protocols are out of the scope of this survey, as we are focusing on CSMA-based LAN protocols.

One of the main purposes of this paper is to identify the main design guidelines that will enable the support of RT communication services in CSMA-based networks, even when the communication environment is shared with uncontrolled traffic sources. As an outcome of this RT communication survey, a RT communication framework is defined, which fulfills the requirements imposed by typical RT applications.

The major challenge concerning the design of protocol architectures for CSMA-based networks is that the channel is a shared resource. Therefore, there is the need to prioritize RT data messages, when the communication infrastructure is shared with external traffic sources [2]. Thus, access to this shared resource needs to be coordinated either centrally or in a distributed manner [3]. According to the ISO/OSI model, this coordination task is performed by the Medium Access Control (MAC) protocols.

Actually, the MAC protocol is the key issue in any broadcast random access network. This paper surveys two of the most relevant random access protocols in wired and wireless environments: the IEEE 802.3 and IEEE 802.11 standard protocols ${ }^{1}$. A common characteristic

\footnotetext{
${ }^{1}$ The protocols defined by these standards are also known as Ethernet and WiFi protocols, respectively.
} 
of these two protocols is the use of the Carrier Sense Multiple Access (CSMA) mechanism to manage the medium access, and their main drawback is the non-determinism of the probabilistic contention resolution algorithm.

Considering the case of wired networks, Ethernet is a well-known and extensively used network technology. The first standardized version was approved and released in 1985 as the ANSI/IEEE 802.3 standard [4]. When Ethernet networks started to be used in process automation plants, simplicity, higher speed and low cost for the communication controllers were the major motivation [5]. However, the traditional shared Ethernet system, with its simple CSMA/CD (CSMA with collision detection) MAC protocol, do not easily allowed the support of RT communication services. Consequently, multiple companies have developed extensions to the Ethernet standards to cover this problem. Today, several systems have fulfilled these specifications, which are known as RT Ethernet. In the last few years, the referred RT Ethernet networks have emerged as the de facto communication standard also for the lower levels of the industrial communication hierarchy [6].

On the other hand, the IEEE 802.11 family of protocols is one of the most used sets of Wireless Local Area Networks (WLANs). It was standardized in 1999 by the IEEE as the 802.11 standard, which was later revised in 2007 [7]. In the last revision, the IEEE 802.11e amendment [8] was incorporated to the original standard. This amendment is intended to provide differentiated levels of Quality of Service (QoS) to the supported applications, including the transport of voice and video over WLANs.

The demand for high performance industrial wireless networking will increase significantly in the next few years. This is a consequence of recent technology developments that demand wireless access in office environments, in public hot-spots and in domestic environments. Presently, there are already big efforts to move from wired to wireless networks [9, 10]. Therefore, it is reasonable to expect that in the near future, the widespread availability of wireless solutions will generate a similar de facto standard for industrial wireless communications.

Within this context, the IEEE 802.11 family of protocols is one of the main contenders to become the de facto standard for industrial wireless communications. However, it has received only limited coverage in this research community [11]. On the one hand, this family of protocols reveals shortcomings in the prioritization of real-time data messages, as the traditional protocol uses a CSMA-based scheme. On the other hand, it is easily able to replace industrial Ethernet solutions in a transparent way, implementing the two lowest layers of the ISO/OSI model, this protocol provides all the required functionalities to enable the support of the Internet Protocol (IP), that is virtually the basis for applications over Ethernet networks.

Traditionally, the RT communication behavior in wired CSMA environments has been guaranteed through the tight control of every communicating device [5]. The coexistence of RT controlled stations with timing unconstrained stations has been made possible by constraining the traffic behavior of the latter. For instance, using traffic smoothers [12, 13].Unfortunately, when moving from wired to wireless networks, the traffic smoothing 
paradigm is no longer adequate, since it is not possible to impose traffic smoothing strategies upon stations that are out of the sphere-of-control ${ }^{2}$ of the RT architecture. Therefore, there is the need to consider the presence of uncontrolled stations when dealing with wireless communications, as the wireless communication medium act as open communication environment. That is, any new participant can try to access the communication medium at any instant (according to the MAC rules) and establish its own communication channels. As a consequence, the system load cannot be predicted at system setup time, nor can be effectively controlled during the system run-time.

It is foreseeable that the RT communication networks will be challenged for moving from closed to open communication environments, and also for partially moving from a wired to a wireless network infrastructure. Thus a new paradigm for RT communications will need to emerge, as the traditional RT communication paradigm is still based on closed and controlled environments. Throughout this paper, it will become clear that the use of WLANs in RT communication domains will challenge the referred paradigm. More accurately, it will be shown that the most promising solutions to support RT communication in CSMA-based networks will be those that allow the coexistence of both RT and non-RT stations in the same communication domain. That is, those solutions that are able to prioritize the RT traffic without the need to control the non-RT communicating devices.

\subsection{Classification Framework}

The state-of-the-art on RT CSMA communications is reviewed in this paper using a classification framework structured in two classification axes (Figure 1). The first axis is related to how collisions are dealt with, in order to provide a RT communication service. Traditional RT communication approaches follow an avoiding collision strategy to guarantee a RT communication service to the supported applications. Another possibility is to replace the traditional probabilistic collision resolution algorithm, in order to deterministically solve the collisions. Finally, it is also possible to enforce the reduction of the number of occurring collisions, through the use of adequate loosely-coupled distributed algorithms. These are the three main classes of the first classification axis.

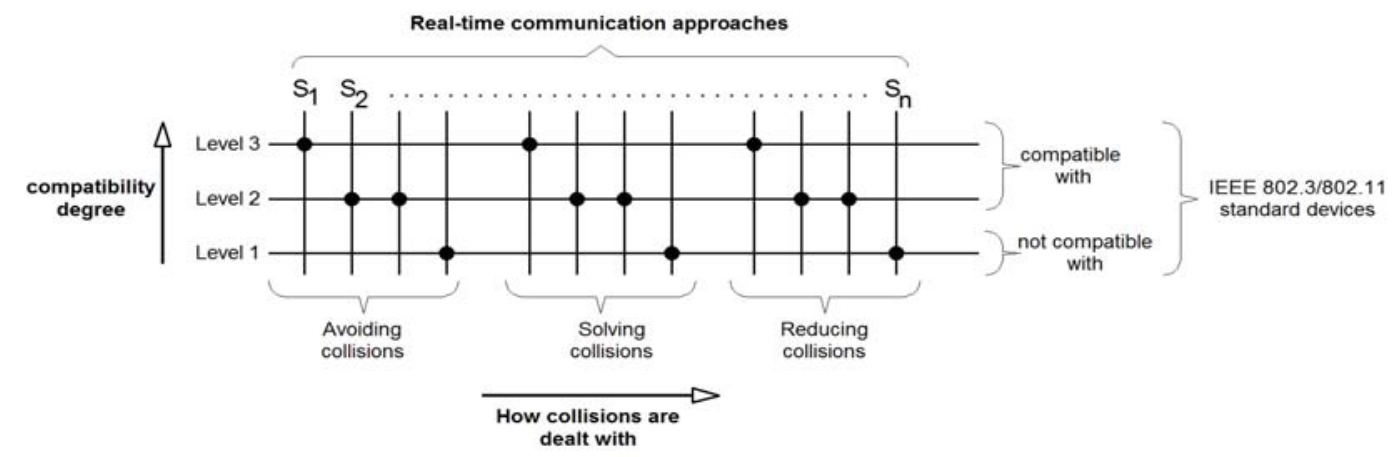

Figure 1. Classification Framework.

\footnotetext{
2 The concept “inside/outside" sphere-of-control was defined by Kopetz [14]. Whenever a RT entity is in the sphere-of-control of a subsystem, it belongs to a subsystem that has the authority to change all the value of this RT entity. Outside its sphere-of-control, the value of the entity can be observed, but cannot be modified.
} 
The second classification axis is related to the compatibility degree with IEEE standard devices. Specifically, this axis highlights how the RT communication approaches keep or alter the compatibility with IEEE 802.3/802.11 compliant devices. Three different compatibility levels have been defined. The level 1 subclass gets together RT communication approaches that require the compliance of all communicating devices with the enhanced (RT) devices. This compliance requirement impairs the use of level 1 devices in open communication environments, as it is unable to handle messages sent by external (uncontrolled) devices. On the other hand, level 2 and level 3 subclasses comprise RT communication approaches able to offer RT guarantees to a subset of enhanced (RT) devices in the presence of default (non-modified) communicating devices. The main difference between these two subclasses is related to the level of modifications required to implement an enhanced (RT) device. The implementation of a level 2 device requires the use of specific hardware, impairing the use of COTS (Commercial Off-the-Shelf) hardware. Conversely, a level 3 device can be implemented upon COTS hardware, requiring just modifications at the firmware/software level of the RT communicating devices. This is an important distinction, as the possibility of using COTS hardware is a relevant advantage when setting-up a RT communication infrastructure.

The remainder of this paper is organized as follows. Sections 2 and 3 describe the state-of-the-art on RT communication in IEEE 802.3 wired networks and IEEE 802.11 wireless networks, respectively. Afterwards, in Section 4 a synthesis of the state-of-the-art is done, where the described RT communication approaches are classified according to the proposed classification axes. One of the main purposes of Section 5 is to identify the most promising RT CSMA-based approaches able to deal with the requirements imposed by next generation communication environments. Specifically, the requirements imposed by a communication medium that is shared with uncontrolled traffic sources. Finally, in Section 6, some conclusions are drawn.

\section{RT Communication in IEEE 802.3 Wired Networks}

The IEEE 802.3 is a widely used network technology, with a MAC protocol based on the Collision Detection among randomly initiated transmissions (CSMA/CD). It has a non-deterministic behavior due to the use of a probabilistic contention algorithm, where the retransmission probability does not depend on the type of traffic, but just on the state of the collision counter of each particular station.

As a consequence, the traditional IEEE 802.3 protocol impairs the support of real-time (RT) communication. Nevertheless, multiple approaches and techniques have been developed to support RT communication services in IEEE 802.3 networks. In this section, a number of relevant research solutions addressing this issue is reviewed.

\subsection{Avoiding collisions}

The first approach, that can be considered when dealing with collisions, is to avoid its occurrence. The most popular solutions are based on the TDMA (Time Division Multiple Access) paradigm. TDMA is a channel access method, which allows multiple nodes to share 
the same channel by dividing the access time into different time slots. Messages are sent at exclusive time slots assigned to each node. It implies a precise clock synchronization among the different nodes so that all nodes are able to agree on their respective transmission slot. One example of a TDMA RT network was proposed by Chen and Lu [15]. This protocol dynamically combines CSMA/CD with a modified version of TDMA, where in the called "under control" state, a NCP (Network Control Packet) message is broadcasted by the control station to synchronize all other stations and to inform them that a new frame started.

A similar approach was proposed by Pritty et al. [16], based on the use of the Timed Packet Release principle, where a monitor node periodically transmits a slot pulse to synchronize the medium access. The monitor node is located at one end of the bus. For each station is allocated a unique time delay, which starts from the receipt of a slot pulse. A station can only start a transmission at the expiration of its time delay, if the bus is silent at that time. Therefore, the value of the time delay assigned to each station is of paramount importance.

Some more recent solutions use Master-Slave techniques, where a special node, the master, instructs the other nodes, the slaves, to transmit at specific time instants. Pedreiras et al. [17] proposed a master/multi-slave transmission control technique called FTT-Ethernet (Flexible Time-Triggered Ethernet) to schedule communications in shared Ethernet networks. In the FTT-Ethernet approach, time is divided in synchronous and asynchronous windows, which are used to, respectively, statically schedule hard RT traffic and dynamically serve soft RT requests (control messages, event-triggered messages and non-RT traffic). The master node implements a centralized scheduler. The distribution of the scheduling decisions to the network stations is periodically performed by the master through a special control message, the trigger message.

Ethernet Powerlink [18] is a commercial master-slave protocol based on the standard IEEE 802.3 layers. Deterministic time is achieved by applying a cyclic timing schedule to all the connected nodes. Each cycle is divided in four distinct phases: start, cyclic, asynchronous and idle periods. In the Start phase, the node management grants the access to the physical medium via the exchange of an explicit frame (SoC - start of cycle) transmitted as a broadcast message to all controllers, thereby preventing collisions. During the Cyclic period, time-critical data is transferred according to a polling request scheme. The Asynchronous phase reserves bandwidth for non time-critical data. The Idle period represents the unused period until the new cycle begins. Any topology can be implemented using hubs. Despite of a recent version that also allows operation over Switched Ethernet networks, the Ethernet Powerlink Standardization Group (EPSG) recommends the use of repeater hubs instead of switching hubs within the RT domains, to minimize path delays and frame jitter.

The Token Passing is other well-known method to avoid collisions in shared broadcast bus networks. This method consists in circulating a token among the stations, where each station is allowed to access the medium only during the token holding intervals. The timed-token protocol [19] is the basis for the RETHER protocol, which was proposed by Venkatramani and Chiueh [20]. This protocol operates in normal CSMA/CD mode until a RT request arrives. Then, it switches to the RETHER mode, where all nodes operate according to 
a token passing protocol. In this mode, time is divided into cycles during which the token regulates the bus access. The token will firstly serve all the RT nodes and then, if there is available time, it will also visit the non-RT nodes. Additionally, every new RT request goes through an admission control system that determines if the request can be satisfied without affecting the existing schedule.

The RT-EP (Real-Time Ethernet Protocol) [21] is also based on an explicit token-passing procedure, where the access to the bus is carried out in two phases: arbitration and application message transmission. In the first, the token visits all the nodes to determine the one holding the highest priority message ready to be transmitted. Then, the token is sent directly to this node for transmission. After concluding the message transmission, the same node starts a new arbitration phase. This means that there is the need for one complete token rotation for the transfer of each single message.

In [22], J. Lee et al. proposed the use of the IEEE 802.4 Token-Passing Bus Access method [23] directly on top of the Ethernet Physical Layer, where a specifically proposed service translator performs the required translation of frame formats and interface functions.

The most recent solutions to support RT-communication in Ethernet networks are based on the IEEE 802.1 Switched Ethernet standard [24]. This standard was introduced in the early 1990s, enables the micro-segmentation of the network by regenerating information only to the receiving port of the Switch. When using Switched Ethernet, it is possible to manage network traffic, by means of the adequate setting of data flow permissions and priorities. The transfer of critical information was addressed both by the IEEE 802.1p and the IEEE 802.1q VLAN [25] standards; the latter extends the priority handling aspects of the 802.1p standard, by providing space in the VLAN (Virtual Local Area Networks) Tag to indicate traffic priorities to support VLANs, while the former gives the ability to prioritize messages.

Finally, it is important to mention that most part of commercial industrial Ethernet solutions (EPA, EtherCAT, Ethernet/IP, Modbus-RTPS, Profinet, Sercos III, TCnet, Vnet) employ full-duplex switches, combined with specific scheduling mechanism at MAC layer. These mechanisms are, in most cases, based on a TDMA, Master-Slave or Token passing approaches. A detailed description of the different Ethernet different solutions can be found in [26].

Nevertheless, it is worth noting that switch-based communications are no longer related to the use of a CSMA-based MAC. Instead, switch-based approaches use a centralized communication scheduler: the switch. Multiple improvements to Switched Ethernet networks have been suggested, mainly to eliminate some of its technological problems [27-29]. An interesting Survey about this issue can be found in [30].

\subsection{Deterministic collision resolution}

The second approach to support RT communications in CSMA-based networks tries to impose a deterministic collision resolution. This can be achieved using one of two different approaches. The first one is by modifying the collision resolution algorithm, in order to guarantee that the colliding frames are serialized in an upper-bounded time interval. The 
second one is by forcing the collision resolution in favor of the RT station, compelling all the other contending stations to abandon the medium access contention.

The CSMA/DCR protocol [31] was one of the first approaches that modify the collision resolution algorithm to deal with RT communication. In the absence of collisions, the CSMA/DCR protocol implements the CSMA/CD access method. Whenever a collision occurs, a binary search tree is used to sort the colliding nodes. A priority hierarchy is enforced, i.e., higher priority nodes try to access the medium prior to the lower priority ones, using an implicit token passing mechanism. Basically, when a collision occurs, the collision period is broken into a previously defined number of time slots. Then, each station can only transmit during its own slot. If a station detects a transmission, it interrupts the slot counting until the end of the transmission. Afterwards, the station waits another interframe space and the slot counting resumes. Consequently, whenever multiple stations want to transmit, they are forced to wait for their assigned slot.

The DOD-CSMA-CD [32] protocol improved the CSMA/DCR protocol, using network station indexes computed on-line, rather than pre-assigned ones. The dynamically computed indices are called time indices. They represent deadline equivalence classes. All messages that are assigned the same index have comparable deadlines. This protocol implements a scheduling algorithm similar to the Earliest Deadline First algorithm (EDF) [33].

In the context of the second approach (forcing the collision resolution), one of the first proposals has been made by Gopal and Wong [34]. In the hybrid control token-CSMA/CD proposal, all stations except the one holding the token work according to the standard CSMA/CD protocol. A station having a packet ready to be transmitted senses the channel and transmits the packet if the channel is sensed idle. If a collision occurs all involved stations terminate their transmissions and schedule retransmissions after a random backoff interval. Conversely, the station that possesses the token gets a higher priority. Specifically, when there is a collision on the channel, the station with the token continues to send data until all other stations are in their backoff phase. Then, the token holding station retransmits its packet immediately (without any backoff interval). The token is implicitly passed to the next station, each time the channel state change from busy to idle. The hybrid control token-CSMA/CD protocol requires specific hardware to be implemented, as it does not comply with the timing behavior defined for IEEE 802.3 standard devices.

The VTPE-hBEB architecture [35] has a similar behavior of the previous described solution. When there is a collision on the channel, the RT station that holds the virtual token acts accordingly to the CSMA/CD protocol: it terminates its transmission and sends a jamming sequence. After the end of the jamming sequence, the RT station starts immediately its transmission, i.e. the backoff interval is equal to zero. Therefore, the VTPE-hBEB architecture complies with the timing behavior defined for IEEE 802.3 standard devices.

Sobrinho and Krishnakumar in [36] designed the EQuB (Ethernet Quality of Service Using Black Bursts) mechanism that also prioritizes the RT traffic by forcing the collision resolution in favor of the RT station. EQuB allows the coexistence of RT and non-RT traffic on the same network domain, providing a bounded access delay to RT packets. The EQuB 
mechanism is based on the assumption that RT stations generate packets in specific intervals, designated as sessions. During a session, RT stations expect to have undisputed access to the medium through the EQuB mechanism. This mechanism works as follows. At the beginning of a session, a RT station works with the conventional Binary Exponential Backoff (BEB) algorithm. However, whenever RT stations participate in a collision, they transmit a jamming sequence up to a pre-specified maximum allowed interval of time in order to avoid that other stations acquire the channel. The jamming signal is called a black burst. The maximum duration of a black burst is a direct function of its contention delay, measured from the time where the access attempt has been scheduled until the host perceives the medium to be idle during an InterFrame Space (IFS). During the transmission of its black burst, a station continuously monitor the channel. If the station detects that no other stations are sending black bursts, it immediately re-initiates the transmission of its packet with success. On the other hand, if the station exhausts its black burst transmission and still senses the bus jammed, it waits for the channel to become idle again (during another IFS) and repeats the algorithm.

Yavatkar et al. [37] proposed the PCSMA (Predictable Carrier Sense Multiple Access) protocol, which is another proposal that follows the paradigm of forcing the collision resolution in favor of the RT station. Under the PCSMA scheme, when a periodic source (RT station) collides with a datagram source (non-RT stations), it does not backoff, but instead continues to transmit. A periodic packet contains enough overhead bits so that a periodic source does not transmit useful data until the collision with the datagram source(s) is resolved.

\subsection{Reducing the number of occurring collisions}

Finally, it is possible to enhance the network responsiveness to RT message requests by reducing the number of occurring collisions. Within this context, Molle and Kleinrock [38] proposed the Virtual Time CSMA (VTCSMA) algorithm. It uses a probabilistic approach combined with specific timing parameters (arrival time, laxity, deadline, length) for the collision resolution. The VTCSMA protocol works as follows. Each station maintains two clocks: a RT clock and a virtual time clock. The virtual time clock runs faster than the RT one. It stops running when the channel is busy, and runs when the channel is idle. The original proposal uses the arrival time to determine when to transmit a message. A message is sent when its arrival time is equal to the time of the virtual time clock. Multiple scheduling policies can be implemented by assigning different waiting times to pending messages. Zhao and Ramamritham [39] presented a performance analysis of four VTCSMA protocols: VTCSMA-A, -T, -D and -L. The VTCSMA-A implements the Molle's original VTCSMA. The VTCSMA-T runs the virtual clock time along the message length axis, where the message with the minimum length is transmitted first. The VTCSMA-D implements the minimum-deadline-first transmission policy and, the VTCSMA-L uses message laxity to determine when to transmit a message. The authors concluded that when the load is very light, collisions are extremely rare. Another relevant result is that the VTCSMA-D achieves the best performance in terms of message loss and collision channel utilization.

The Window Protocol [40] implements a dynamic time window to reduce the number of 
occurring collisions. In this case, all stations continuously monitor the channel, and after every successful message transmission, all stations select an initial time window. Once an initial time window has been selected, if just one station has a message ready to be transmitted and the message is within the window, then it will be sent. If several stations have messages to be transmitted within the window, the window size is reduced until there is just one remaining message; if there are no nodes with messages within the window, the window size can be increased.

In the Window protocol all stations must follow the same policy to select the initial time window, as well as to update the time window whenever a collision occurs. In [41, 42], Kurose et al. proposed a Window protocol implementing the minimum-laxity-first (MLF) policy. They assume that the laxity of all messages are constant. Zhao et al. [39] suggested a Window protocol implementing the latest time to send policy (LS). Furthermore, a newly arriving message is immediately considered for transmission, if its LS is smaller than those of all pending messages in the system.

In [43], the authors presented a modified CSMA/CD protocol, called the Dynamic pi-persistent CSMA/CD protocol. The pi-persistent protocol is a variant of the window protocol, where the transmission probability of a ready packet depends on its laxity, and a time window is used to reduce the number of collisions in heavily loaded scenarios.

Finally, the traffic smoothing mechanisms proposed by Kweon et al. [12, 44] constrain the packet generation rate of non-RT messages below a defined threshold, in order to provide a probabilistic guarantee of message delivery. The original traffic smoothing proposal uses the well-known leaky bucket regulator [45]. The leaky bucket has two parameters: the bucket size (BS) and the refresh period (RP). The BS bounds the maximum number of credits that can be stored in the credit bucket. If a packet arrives when the bucket is full, the packet is discarded. When a packet arrives and there is no credit in the bucket, the packet is kept in the queue until new credits arrive. The credits are added to the bucket every RP period. Packets are sent into the network at constant transmission rate, thus smoothing traffic bursts.

Several policies for traffic smoothing have been proposed. The HIMD (Harmonic-Increase and Multiplicative Decrease) [44] is a dynamic policy that uses the credit bucket depth and the refresh period as a dynamic traffic regulator; in the absence of collisions, it periodically increases the input bound by periodically reducing the refresh period. In [13], the smoothing actions are performed by a fuzzy controller, where the network load is observed along determined time intervals, via the measurement of the throughput and of the number of occurring collisions.

\section{RT Communication in IEEE 802.11 Wireless Networks}

Both wired IEEE 802.3 and wireless IEEE 802.11 networks use the CSMA algorithm to manage the medium access. The main difference between both approaches is that in the case of wireless environments the Collision Detection procedure (CSMA/CD) cannot be used, as it would require the implementation of a full-duplex radio. As an alternative to the CSMA/CD protocol, the IEEE 802.11 standard [7] implements a Collision Avoidance procedure 
(CSMA/CA), that is referred as the Distributed Coordination Function (DCF).

In the following subsections, a number of relevant solutions to support real-time (RT) communication in IEEE 802.11 wireless networks are described, according to the same classification axis that was used in the case of wired IEEE 802.3 networks.

\subsection{Avoiding collisions}

The Point Coordination Function (PCF) is one of the main solutions intended to avoid collisions in IEEE 802.11 wireless networks. It has been proposed in the original IEEE 802.11 standard [7] as an optional access mechanism. It implements a centralized polling scheme to support synchronous data transmissions, where the Point Coordinator (PC) performs the role of polling master. When the PCF scheme is used, the time scale is divided in two super-frames consisting of a Contention Period (CP), used by the DCF scheme and a Contention Free Period (CFP), used by the PCF.

The IEEE 802.11e amendment [8] incorporates an additional coordination function called hybrid coordination function (HCF), that is only used in QoS network configurations. The HCF provides two new mechanisms: the Enhanced Distributed Channel Access (EDCA), which delivers traffic based on differentiating user priorities (UPs) and; the Hybrid Coordination Function (HCF) Controlled Channel Access (HCCA), which allows the reservation of transmission opportunities (TXOPs) with the hybrid coordinator (HC).

The HCCA mechanism was proposed to improve the PCF scheme. It is intended to guarantee bounded delay requirements, based on a Round Robin scheme. In contrast to the PCF scheme included in the legacy 802.11 MAC, the HCCA operates during both the CFP and CP periods (Figure 2).

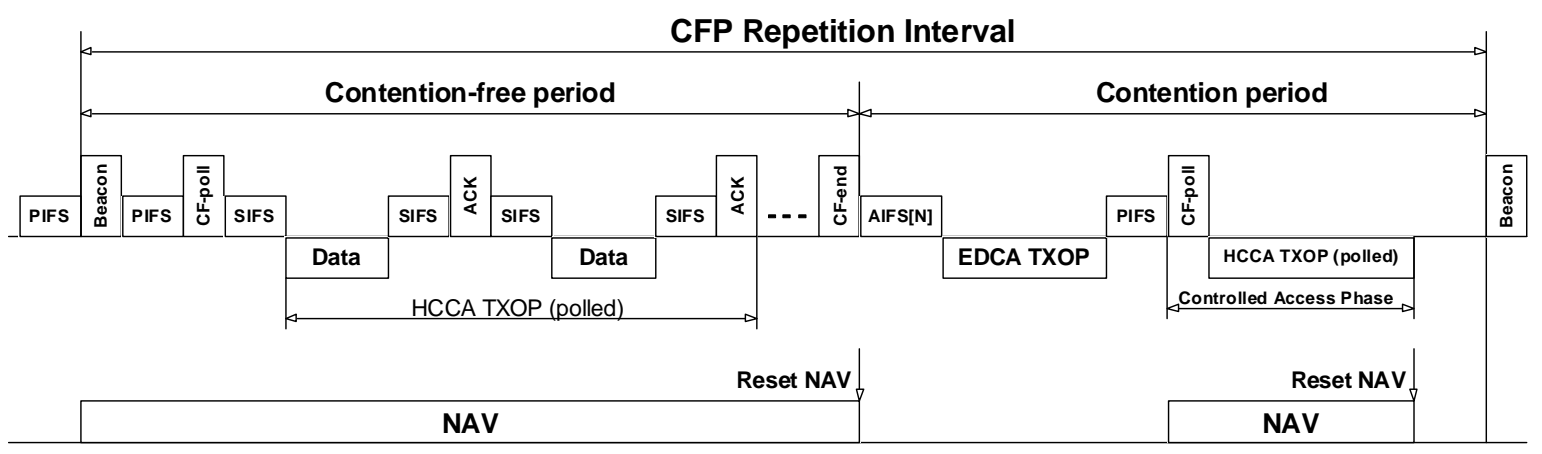

Figure 2: Example of CFP repetition interval.

The HC gains control of the wireless medium by waiting a shorter time between transmissions than the stations using the EDCA or DCF procedures. The HC may include a CF (Contention Free) parameter set element in the Beacon frame, in order that all stations set their NAVs (Network Allocation Vectors) to the end of the controlled phase. During the CFP, the HC controls the access to the channel by polling all the stations in the polling list. To each polled station is granted a transmission opportunity (TXOP). On the other hand, it is also allowed that the HC starts a TXOP during the CP. 
Similarly to the PCF scheme, the HC also polls all the stations in the polling list, even though some stations may have no messages to transmit. When the HC polls a station that has no packets to transfer, the station will transmit a null frame, after the QoS CF-poll. As a consequence, the polling overhead is roughly equal to the time interval from sending the polling frame till the end of the ACK frame [46].

A number of improvements has been proposed to reduce the HCCA polling overhead. For instance, Gao et al. [47] proposed a new admission control framework to replace the traditional mechanism that use the mean data rate and the mean packet size to calculate the resource needed by a flow. Then, this new mechanism is adequate specially when dealing with variable bit rate (VBR) traffic over HCCA.

Rashid et al. [48] have pointed out the performance deficiencies of the HCCA scheduler and, they proposed a new scheduling scheme, namely, the prediction and optimization-based HCCA (PRO-HCCA), which use a prediction mechanism to account for the dynamic intensity of VBR traffic. This mechanism try to find an optimal allocation of available transmission time among the competing traffic streams.

Son et al. [46] proposed a polling scheme where the HC punishes the stations that have no packets to transmit. When a station transmits a null frame, this station will not be polled again during a period of time.

Lo, Lee and Chen [49] designed a multipolling mechanism called Contention Period Multipoll (CP-Multipoll), which incorporates the DCF access scheme into the polling scheme. It uses different backoff values for the multiple message streams in the polling group, where each station executes the backoff procedure after receiving the CP-Multipoll frame. The contending order of these stations is the same as the ascending order of the assigned backoff values.

In [50], it is proposed a scheduling algorithm referred as ARROW (Adaptive Resource Reservation Over WLANs), it allocates the available bandwidth based on the actual amount of data awaiting transmission in every station. ARROW exploits the Queue Size field, introduced by IEEE 802.11e as part of the new QoS Data frames. This field can be used by the QoS stations to indicate the amount of buffered traffic for their traffic specifications. Therefore, the proposed scheduler utilizes this information to allocate TXOPs to QOS stations in such a way that satisfies these transmission requirements, as long as they comply with the traffic specifications. This is in contrast to other proposals, which perform channel allocations based on the estimated buffered data in every station.

Lee et al. [51] proposed a polling scheme based on a master slave solution. A virtual polling list (VPL) contains the MAC address of the wireless slaves to be polled, and a virtual polling period (VPP) defines the duration of the polling cycle. When a slave receives a poll frame from the master, it can transmit a response frame to the master, or directly to another slave. Furthermore, after polling all the slaves registered in the VPL, the master invites other slaves into the network through the broadcast of an entry claim frame.

Solutions based on token passing mechanisms have also been proposed. In [52], Ergen et 
al. proposed the WTRP (Wireless Token Ring Protocol), which is a MAC protocol that exchanges special tokens and uses multiple timers to maintain the nodes synchronized. The token is rotated around the ring. Each station transmits during a specified time and if enough time is left, the station invites nodes outside the ring to join. Cheng et al. [53] presents a wireless token-passing protocol, named Ripple. Basically, Ripple modifies the data transmission procedure of 802.11 DCF and employs request-to-send (RTS) and ready-to-receive (RTR) frames as tokens. A node that has the right to send a data frame will send a RTS frame, and a node which has the right to receive a data frame will send a RTR frame to the sender if the expected RTS frame has not been received. Summing up, a station can only send a DATA frame if it holds the token.

In [54], Willig presented the FTDMA (Flexible TDMA) MAC protocol. FTDMA is based on a polling scheme, where a base station polls all registered RT stations in every frame. A frame is logically subdivided into phases: SYNC, Polling, Reservation, Register, Current Scheduler and Data Transfer. The main advantage of the FTDMA over traditional TDMA solutions is that unused slots can be used by other stations.

In [55], Miorandi et al. proposed a solution based on a Master-Slave architecture on top of IEEE 802.11. In that proposal, cyclic packets are exchanged by means of periodic queries sent by the master to the slaves. Three different techniques were proposed to handle acyclic traffic: the first technique queries the slaves that signaled the presence of acyclic data, at the end of the current polling cycle. The second technique allows a slave, when polled, to send directly acyclic data to the master. The third one exploits the decentralized nature of the IEEE 802.11 MAC protocol. When acyclic data is generated, it allows a slave to immediately try to send data to the master.

\subsection{Deterministic collision resolution}

Another approach to support Qos guarantees are those based on forcing the collision resolution schemes in favor of the RT stations. A relevant proposal has been made by Sobrinho and Krishnakumar [56], who adapted the EQuB mechanism (black burst) [36] to ad hoc CSMA wireless networks. This scheme requires the shutdown of the standard retransmission scheme. Real-time stations implementing the EQuB approach contend for the channel access after a medium interframe spacing tmed, rather than after the long interframe spacing tlong, used by standard stations. Thus, RT stations have priority over standard stations. When a RT station wants to transmit, it sorts its access rights by jamming the channel with Black Bursts (BB's), i.e., energy pulses immediately after an idle period of length tmed. The length of the BB transmitted by a RT node is an increasing function of the contention delay experienced by the node.

A similar scheme is presented in [57], where voice nodes (RT stations) use energy-burst (EB) (that are similar to $\mathrm{BB}$ ) periods to prioritize RT packets over data packets. The AP (Access Point) can transmit a VoIP packet after PIFS without backoff or contention. On the other hand, each voice station has its own address (ID), referred as VID (virtual identification). The VID can be assigned during the traffic stream (TS) setup procedure. The VID is expressed as a binary value, which is determined by the voice packet resolution period 
(VPRP). The station with the highest VID wins the contention.

In [58], Sheu et al. proposed a priority MAC protocol based on Sobrinho's approach, complemented by a binary tree referred as contention tree. Basically, the black-burst scheme is adopted to distinguish the priorities of stations. Stations with the same priority send messages in a round robin manner. The basic idea is that a station can obtain an unique ID number, which depends on its position in the contention tree.

In [59], a RT-communication approach (VTP-CSMA) has been proposed based on a traffic separation mechanism. Such mechanisms are able to prioritize RT-traffic over other traffic, without directly controlling the latter. The proposed architecture is based on a Virtual Token Passing procedure that circulates a virtual token among a number of RT devices. This virtual token is complemented by an underlying traffic separation mechanism that prioritizes the RT traffic over the non-RT traffic.

The above underlying traffic separation mechanism has a similar behavior to both the SVP protocol [60] and the mechanism proposed by Hwang and Cho [61]. The SVP protocol specifies a backoff value of zero for stations or classes with the highest priority level. A shortcoming of this mechanism is that if multiple SVP stations attempt to transmit at the same time, consecutive collisions will occur and a failure will be reported. The mechanism proposed in [61] consists in allowing the transmission of voice packets (highest priority) in the first empty slot in the first retransmission. When the first retransmission fails, the second retransmission performs the original backoff procedure. Therefore, these approaches are not able by themselves to provide RT guarantees to the supported applications.

\subsection{Reducing the number of occurring collisions}

The EDCA mechanism available in IEEE 802.11e standard is specifically intended to reduce the number of occurring collisions. Its underlying idea was previously proposed by Deng and Chang [62], where the higher priority class uses the window $\left[0,2^{j+1}-1\right]$ and the lower priority class uses the window $\left[2^{j+1}, 2^{j+2}-1\right]$, where $j$ is the backoff stage.

The EDCA function implements a CSMA/CA mechanism for the channel access under the control of the HCF coordination function. It enhances the DCF scheme, as each frame arriving at the MAC layer with a defined priority will be mapped into one of the four access categories (AC). Different levels of service are provided to each of the AC traffics, based on three independent mechanisms: (i) the Arbitration Interframe Space (AIFS); (ii) the TXOP time interval and; (iii) the Contention Window size (CW). Firstly, for a station operating under EDCA, a frame will be transferred only if the channel remains idle during an AIFS[AC] interval. This means that the duration of the interframe space is related to the access category. Figure 3 shows the relationships between the multiple AIFSs in the EDCA scheme. 


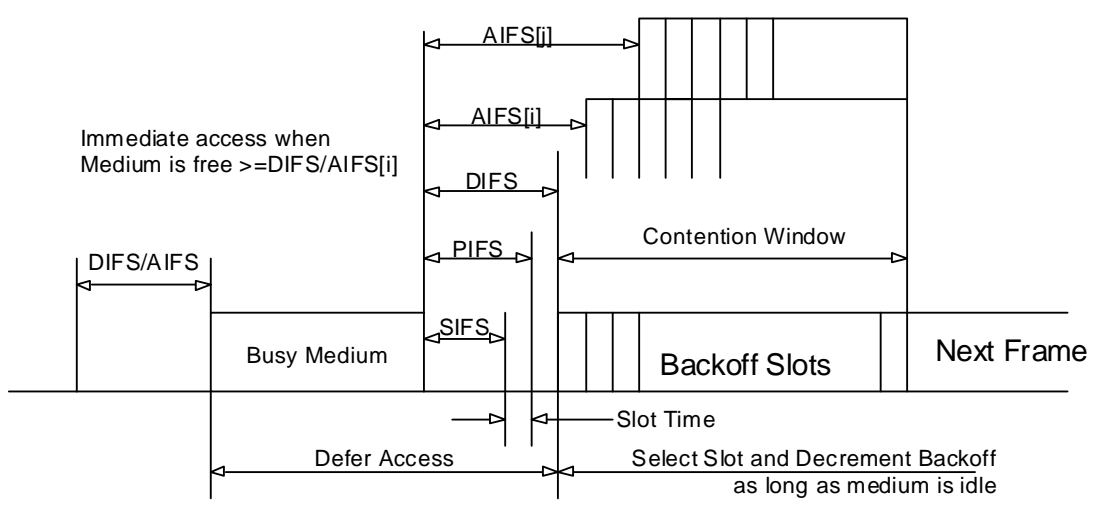

Figure 3: Interframe spaces in the EDCA mechanism.

The EDCA mechanism also introduced the TXOP concept that is the time interval during which the station keeps the medium access control. Consequently, multiple frames may be transmitted within an acquired TXOP, if there is more than one frame pending to be transferred in the AC for which the channel has been acquired.

Finally, if a station wants to transmit a frame while the channel is busy, or becomes busy before the expiration of the AIFS[AC], the backoff procedure is invoked (third traffic differentiation mechanism). The contention window is defined by the $a C W_{\min }[A C]$ and $a C W_{\max }[A C]$ attributes, in contrast to the legacy DCF where the initial values were randomly selected among the $[0, C W]$ interval defined by the physical layer. In the EDCA mechanism, the backoff procedure selects a random number, in the range $[0, C W]$, where the $C W$ size is initialized at $a C W_{\min }[A C]$. When a transmission fails, $C W$ is increased by $[($ oldCW $[A C]+1) * P F-1]$ upper bounded by $a C W_{\max }[A C]$ where $P F$ is the persistence factor (its default value is $P F=2$ ). On the other hand, the backoff counter decreases the backoff interval whenever the medium is detected to be idle for AIFS[AC].

As the EDCA mechanism provides 4 access categories, it would be expectable that the highest access category (voice) would be adequate to transfer RT messages. In a previous research work [63], the authors have assessed the behavior of this category when used to transfer periodic small sized packets in an open communication environment. Both the number of packet losses and the average size of the MAC queues forecast an unacceptable number of deadlines losses for RT message streams, even for intermediate load cases. Therefore, additional mechanisms must be provided in order to adequately support RT communication.

Based on the EDCA mechanism, Villalón et al. [64] designed the B-EDCA mechanism that intends to provide QoS support to multimedia communication, and it is able to coexist with legacy DCF-based stations. Basically, it changes the AIFS value of the highest AC to SIFS+aSlotTime when stations are in the Backoff state. Moreover, in order to keep the compatibility with the HCCA mechanism, a stations implementing the B-EDCA mechanism must wait for an additional SIFS time when the backoff counter reaches zero, i.e. $2 \times$ SIFS + aSlotTime. Therefore, it is adequate when operating in the IEEE $802.11 b$ PHY mode, 
as the waiting time will be larger than the one used by HCCA mechanism and smaller than the time used by standard EDCA stations.

In [65], it is also proposed a modified EDCA mechanism, where soft real-time guarantees constraints is guaranteed by dynamically adjusting the priority level of a traffic flow based on the estimated per-hop delay, and generating a non-uniformly distributed backoff timer for retransmitted frames according to their individual end-to-end delay requirements.

Hamidian and Körner [66] presented an interesting solution that allows stations with higher priority traffic to reserve time for collision-free access to the medium. Basically, it proposes the transfer of the HCCA admission control and the scheduling algorithms from the HCCA controller to the contending stations. It uses the traffic specification (TSPEC) as defined in the draft version of the IEEE 802.11e HCCA standard [67]. The TSPEC is an element sent through a management frame that contains information about the characteristics and QoS expectation of a traffic stream. For instance, the maximum service interval specifies the maximum time interval between the start of two consecutive service periods. The scheduling and the admission control of a new traffic stream is locally made at each station.

Wang et al. [68] designed a new collision resolution mechanism, referred as gentle DCF or GDCF. The difference between GDCF and DCF is that GDCF takes a more conservative measure by halving the $\mathrm{CW}$ (Contention Window) value only if there are $c$ consecutive successful transmissions. Conversely, DCF reset its CW to the minimum value once there is a successful transmission.

Yang and Vaidya [69] proposed the Busy Tone Priority Scheduling (BTPS) protocol. BTPS works similarly to the IEEE 802.11 DCF, with the difference that high priority and low priority behave differently during IFS and backoff stages. The BTPS protocol uses DIFS as the IFS for high priority stations. However, during DIFS and backoff stages, the high priority stations with queued packets send a BT1 (busy tone) pulse every $M$ slots, where $M$ is a constant. Between two consecutive busy tone pulse transmissions, there should be at least one empty SlotTime interval as the station must have a chance to listen to the data channel. Therefore, $M$ could be any value larger than or equal to 2 and, the IFS of low priority stations should be larger than M slots, in order to enable sensing the busy tone signal.

In [70], it was proposed a distributed algorithm intended to provide fair scheduling in a wireless LAN, referred as DFS (Distributed Fair Scheduling). The DFS protocol behaves quite similarly to IEEE 802.11 DCF, except in what concerns the backoff interval that is initially calculated. The fundamental difference is that each station maintains a local virtual clock and, the backoff interval is chosen proportionally to the finish tag of the packet to be transmitted. The finish tag is calculated similarly to the SCFQ (Self-Clocked Fair Queueing) algorithm [71]. In [72], the authors modify the distributed SCFQ algorithm combined with the prioritization schemes proposed in the EDCA mechanism and specify the RT-FCR (real-time fast collision resolution), where the priorities are implemented by assigning different backoff ranges based on the type of traffic.

In [73], Lopez-Aguilera et al. evaluated the performance of the IEEE 802.11e EDCA 
when its working procedure is unsynchronized. The authors proposed the use of AIFS times values that are separated by values that are not multiple of the slot time. As a consequence, it would become possible to avoid collisions between frames from different access categories.

Lo Bello et al. [74] proposed a wireless traffic smoother (WTS) to support soft RT traffic over IEEE 802.11 WLANs. The presented solution is similar to the traffic smoother scheme previously proposed for Ethernet networks [13]. However, its main drawback is that it requires the smoothing strategy to be implemented in all the communicating devices.

\section{Synthesis of the State-of-the-Art}

In the previous sections, some of the most relevant CSMA-based real-time (RT) communication proposals have been presented according to how collisions are dealt with. In this section, those RT communication proposals are now classified according to a compatibility degree axis. This second classification axis highlights how such proposals keep or alter the compatibility with IEEE 802.3/802.11 compliant devices.

As previously explained (Section 1.1), three different compatibility levels are defined. Level 1 proposals impair the coexistence between enhanced (RT) devices and default (non-modified) devices in the same network domain. On the other hand, level 2 and level 3 subclasses get together the RT communication proposals able to offer RT guarantees in presence of default (non-modified) devices. The main difference among these two subclasses is related to the required level of modifications. The implementation of a level 2 device requires the use of specific hardware, impairing the use of COTS (commercial off-the-shelf) hardware. Conversely, a level 3 device can be implemented upon COTS hardware, requiring just modifications at the firmware/software level of the RT communicating devices.

Figures 4 and 5 classify the reported RT communication proposals according to these two classification axes, for wired IEEE 802.3 and wireless IEEE 802.11 networks, respectively. When dealing with the next generation communication environments, it is of utmost importance the coexistence between RT stations and third stations (stations that are out of the sphere-of-control of the RT architecture). Therefore, approaches with compatibility degree level 1 are not adequate, as they are not able to handle messages sent by external (uncontrolled) devices. As a consequence, the ability to support next generation communication environments will strongly rely upon technical solutions with compatibility degree level 2, being desirable that solutions of level 3 arise. 


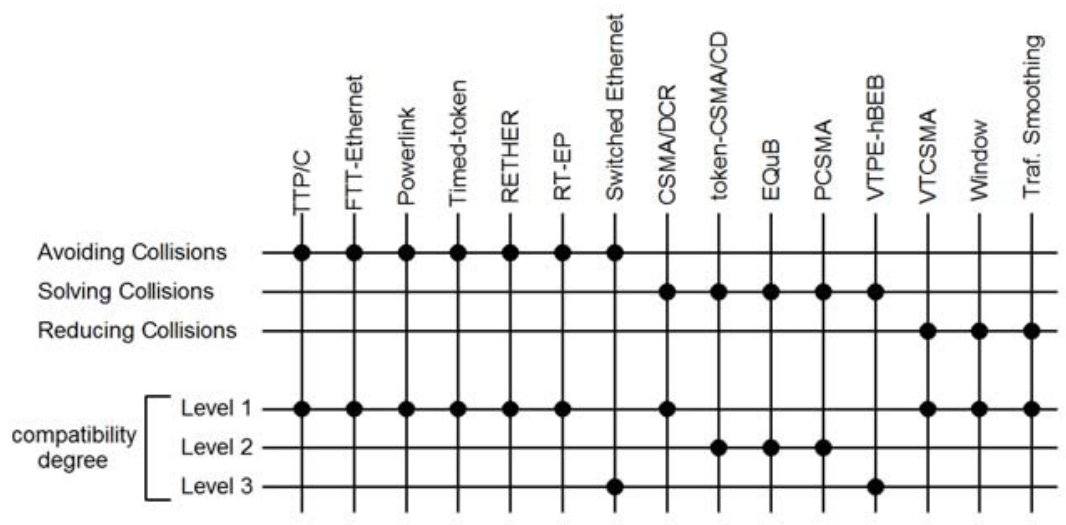

Figure 4: Supporting RT communication in IEEE 802.3.

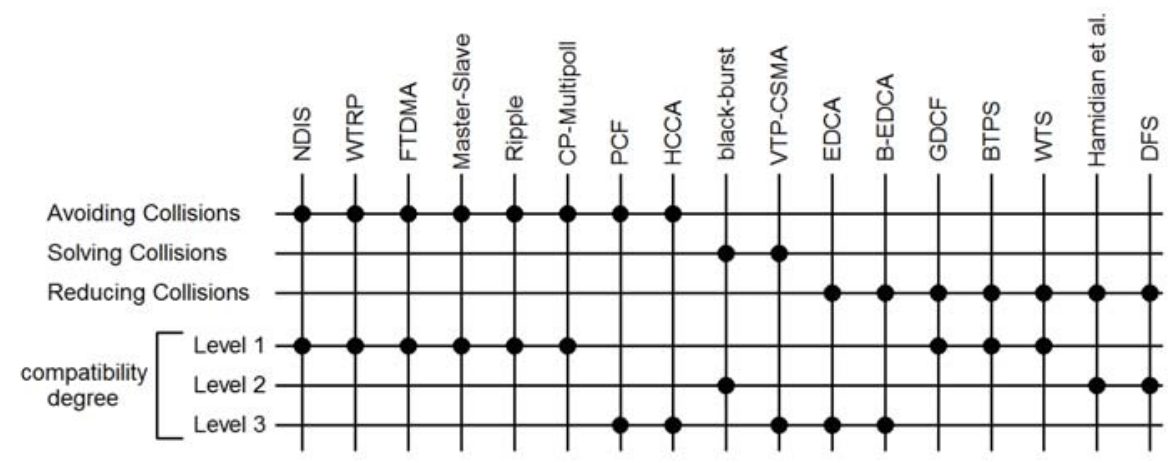

Figure 5: Supporting RT communication in IEEE 802.11.

Most part of the RT solutions that follow the avoiding collisions strategy are based on TDMA, Token-Passing, Master-Slave or Polling techniques. A common characteristic of these RT solutions is that a enhanced (RT) station is not able to support RT communication in the presence of default (non-modified) IEEE 802.3/802.11 stations (unless these default stations do not initiate any communication). That is, the majority of the avoiding collision solutions have a compatibility degree level 1. Relevant exceptions are: the Switched Ethernet solutions and the improvements included in the HCF (PCF and HCCA) mechanism of the IEEE 802.11e amendment. However, the Switched Ethernet violates the decentralized paradigm of the CSMA protocol. The Switched Ethernet enables the micro-segmentation of the network, thus each station operates in a Carrier Sense Single Access method. Therefore, the Switched Ethernet approach does not really follows a CSMA-based scheme.

Additionally, regarding the IEEE 802.11 standard approaches, most part of the WLAN network cards never actually implemented the PCF scheme, due to complexity reasons [75]. Therefore, the PCF mechanism has not been a solution to support RT communication, due to the unavailability of WLAN network cards. The HCCA mechanism [8] has been proposed as an improvement to the PCF mechanism. However, some studies [47, 48] have already shown that the HCCA mechanism may not be suitable to guarantee the special requirements of RT applications. In order to overcome drawbacks of the scheduler and admission control of this mechanism, a number of improvements have been proposed, as mentioned in subsection 3.1. 
But, it is still not clear whether the HCCA mechanism will be implemented in the next generation WLAN network cards, overcoming the unavailability problem of the PCF mechanism.

Besides these two approaches, all other avoiding collision approaches require the strict control of every communicating entity. Otherwise, they are not able to work properly, as they are not able to handle messages sent by external (uncontrolled) stations.

Concerning the second sub-class (solving collisions), several Level 2 and Level 3 techniques are based on the black-burst scheme (EQuB and $\mathrm{BB}$ ) and the Virtual Token approaches (VTPE-hBEB, Hybrid token-CSMA/CD, PCSMA and VTP-CSMA). These kind of solutions implement the paradigm of forcing the collision resolution in favor of the RT stations. To our best knowledge, this type of medium access technique is the only one that allows the coexistence of RT stations with external uncontrolled traffic sources, being able to prioritize the RT communication.

Analyzing the specific forcing-collision based approaches presented in this paper, the main drawback of the hybrid token-CSMA/CD mechanism is that it requires the stations to be synchronized. Besides, this mechanism is also not compatible with COTS Ethernet hardware. Conversely, the EQuB is a very interesting technique that enables a privileged access to RT traffic with a FCFS (First-Come-First-Serve) discipline. Another interesting approach is the PCSMA protocol, which adds extra bits to the RT packet. When a collision with non-RT packet occurs, such extra bits guarantee that it has not been transmitted useful data before the collision resolution.

In the case of wireless networks, there are some adaptations of the black-burst scheme for forcing the collision resolution in favor of RT station. The VTP-CSMA architecture may be compared with the black-burst scheme. The main disadvantage of the BB scheme is that it compels the modification of the MAC layer and possibly also of parts of the PHY layer (e.g. radio, ICs), which impairs the use of COTS hardware. Although VTP-CSMA mechanism also needs to modify parts of the MAC layer, it could be implemented in COTS hardware (e.g. FPGA) upon standard 802.11 hardware.

Finally, concerning the last subclass (reducing collisions), it is worthwhile to mention the solutions that constrain the generated traffic in a fair way, without imposing any further modification to the MAC protocol. Solutions like [12, 38-40, 43, 44, 64] increase the network access fairness and reduce the number of collisions based on some priority criterion. The main drawback of all these solutions is the compatibility degree level 1. For instance, the traffic smoothing approach requires the smoothing strategy to be implemented in all the communicating devices. Therefore, it is not able to cope with external (uncontrolled) traffic sources.

\section{Real-time framework for CSMA-based networks}

We entirely agree with Bianchi et al. [76] that in wireless architectures, the service differentiation mechanism must be compulsory introduced as medium access control (MAC) layer extension and analyzing the state-of-the-art approaches described in this paper, we may 
conclude that: The most promising solutions to prioritize RT communication in CSMA-based networks are those that force the collision resolution in favor of the RT stations, compelling all the other contending stations to postpone the medium access. That is, all those solutions based on a "Forcing Collision Resolution" (FCR) paradigm. Unfortunately, most part of FCR-based approaches are just able to prioritize the RT traffic. This means that, whenever two or more RT stations simultaneously contend for the medium access, the FCR MAC will not be able to serialize the contending stations. Therefore, there is also the need to coordinate the medium access among the multiple RT stations.

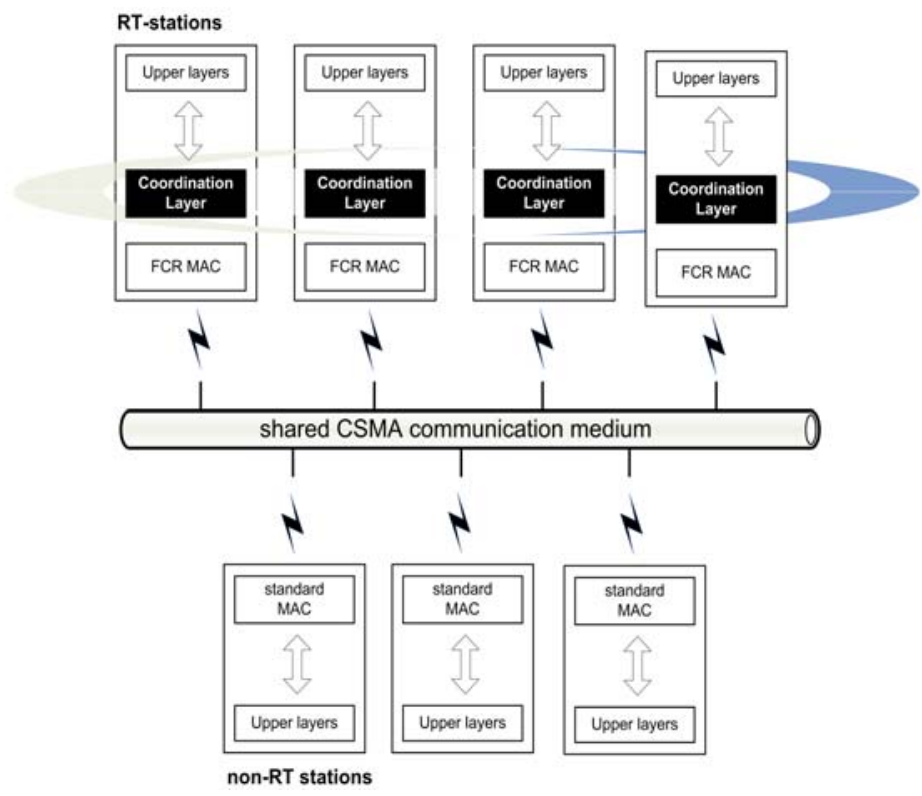

Figure 6: A 2-tier architecture to support real-time communication in wireless networks.

Within this context, we consider that a 2-tier architecture (Figure 6) is an adequate architecture to support RT communication in CSMA-based shared communication environments. This architecture encompasses: (a) in the lower layer (MAC layer), a forcing collision resolution (FCR) mechanism that enforces a high priority level access to the subset of RT stations; (b) in the upper layer, a coordination mechanism that ensures a collision-free access among the subset of RT stations. This coordination mechanism can be based on, for instance, a token passing scheme or a time division multiple access (TDMA) mechanism. It must serialize the medium access of just the RT stations, as the underlying FCR layer ensures that non-RT stations will always loose the medium contention when contending with a RT-station.

\section{Conclusions}

Ethernet and WiFi are well-known and extensively used network technologies. However, one of their main disadvantages is the inherent non-determinism of its probabilistic contention resolution mechanism. This probabilistic behavior impairs both communication protocols to provide a real-time (RT) communication service to the supported applications, unless additional functionalities are introduced.

On the one hand, there are several techniques to support RT communications for Ethernet 
networks. However, few of those techniques allow default (non-modified) devices to coexist with modified (real-time) stations in the same communication environment (compatibility levels 2 and 3). Thus, all the network stations must be under the strict control of the RT communication architecture (compatibility level 1). Otherwise, the RT-communication will be highly disturbed.

On the other hand, there is a trend for the implementation of RT communication systems on top of wireless networks, and, specifically, on top of WLAN [9, 10, 77]. A fundamental assumption that must be considered is that the wireless communication medium is an open communication environment, i.e., any new participant can try to access the communication medium at any instant and establish its own communication channels. Therefore, the traditional RT communication approaches that guarantee RT behavior through the tight control of every communicating device (compatibility level 1) are no longer applicable.

Summing up, the coexistence of default (non-modified) devices together with modified (real-time) devices is a hard task. Specially in the case of wireless networks due to the open characteristics of the communication medium. Therefore, the underlying communication protocols must be able to guarantee the timing constraints of the RT traffic in a communication medium that is potentially being shared with external (uncontrolled) traffic sources. Thus, state-of-the-art approaches that do not allow the coexistence of both RT and non-RT stations will not be able to handle next generation communication scenarios. As a consequence, whatever the RT communication solution, it is mandatory to define a lower communication layer that enables the separation of the RT and the non-RT traffic in any shared communication environment.

\section{References}

[1] P. Ramanathan, "Overload management in real-time control applications using (m, k)-firm guarantee," IEEE Transactions on Parallel and Distributed Systems, vol. 10, pp. 549 - 559, 1999.

[2] T. Sauter and F. Vasques, "Editorial: Special Section on Communication in Automation," IEEE Transactions on Industrial Informatics, vol. 2, pp. 73 - 77, 2006.

[3] B. Tavli and W. Heinzelman, Mobile Ad Hoc Networks. Dordrechtf: Springer, 2006.

[4] "IEEE Standard for Information Technology - Telecommunications and information exchange between systems - local and metropolitan area networks - specific requirements. Part 3: Carrier Sense Multiple Access with Collision Detection (CSMA/CD) access method and physical layer specifications," ANSI/IEEE Std 802.3, 1985.

[5] J.-D. Decotignie, "Ethernet-based real-time and industrial communications," Proceedings of the IEEE, vol. 93, pp. 1102 - 1117, 2005.

[6] M. Felser, "Real-time Ethernet - Industry prospective," Proceedings of the IEEE, vol. 93, pp. 1118 - 1129, 2005.

[7] "IEEE Standard for Information Technology - Telecommunications and information 
exchange between systems -local and metropolitan area networks-specific requirements - part 11: Wireless LAN Medium Access Control (MAC) and Physical Layer (PHY) Specifications," IEEE Std 802.11-2007 (Revision of IEEE 802.11-1999), 2007.

[8] "IEEE Standard for Information Technology - Wireless LAN Medium Access Control (MAC) and Physical Layer (PHY) Specifications Amendment 8: Medium Access Control (MAC) Quality of Service Enhancements," IEEE Std 802.11e-2005, 2005.

[9] A. Willig, K. Matheus, and A. Wolisz, "Wireless technology in industrial networks," Proceedings of the IEEE, vol. 93, pp. 1130 - 1151, 2005.

[10] M. Jonsson and K. Kunert, "Towards Reliable Wireless Industrial Communication With Real-Time Guarantees," IEEE Transactions on Industrial Informatics, vol. 5, pp. $429-442,2009$.

[11] A. Willig, "Recent and Emerging Topics in Wireless Industrial Communications: A Selection," IEEE Transactions on Industrial Informatics, vol. 4, pp. 102-124, 2008.

[12] S.-K. Kweon and K. G. Shin, "Statistical real-time communication over Ethernet," IEEE Transactions on Parallel and Distributed Systems, vol. 14, pp. 322-335, 2003.

[13] L. Lo Bello, G. A. Kaczynski, and O. Mirabella, "Improving the real-time behavior of Ethernet networks using traffic smoothing," IEEE Transactions on Industrial Informatics, vol. 1, pp. 151-161, 2005.

[14] H. Kopetz, "Time-triggered model of computation," Real-Time Systems Symposium, pp. 168 - 177, 1998.

[15] W.-H. Chen and C.-C. Lu, "CSMA/CD/TDMA: A dynamic combination for voice and data integration," Proceedings - IEEE INFOCOM, pp. 842 - 849, 1990.

[16] D. W. Pritty, J. R. Malone, D. N. Smeed, S. K. Banerjee, and N. L. Lawrie, "Real-time upgrade for Ethernet based factory networking," IECON Proceedings (Industrial Electronics Conference), vol. 2, pp. 1631 - 1637, 1995.

[17] P. Pedreiras, P. Gai, L. Almeida, and G. C. Buttazzo, "FTT-Ethernet: A flexible real-time communication protocol that supports dynamic QoS management on Ethernet-based systems," IEEE Transactions on Industrial Informatics, vol. 1, pp. 162 - 172, 2005.

[18] "Ethernet Powerlink protocol," Available at http://www.ethernet-powerlink.org.

[19] R. M. Grow, "A timed token protocol for local area networks," Electro/82 Conference Record, pp. 17 - 3, 1982.

[20] C. Venkatramani and T.-C. Chiueh, "Supporting real-time traffic on Ethernet," Real-Time Systems Symposium (RTSS), pp. 282 - 286, 1994.

[21] J. M. Martinez and M. G. Harbour, "RT-EP: A fixed-priority real time communication protocol over standard Ethernet," 10th Ada-Europe International Conference on Reliable Software Technologies (Lecture Notes in Computer Science), vol. 3555, pp. 180 - 195, 2005.

[22] J. Y. Lee, H. J. Moon, S. Moon, K. W, S. Lee, and I. Park, "Token Passing Bus Access Method on the IEEE 802.3 Physical Layer for Distributed Control Networks," 15th IFAC Workshop on Distributed Computer Control Systems, 1998.

[23] "IEEE Standard for Information Technology - "Information Processing Systems - 
Local Area Networks - Part 4: Token-passing bus access method and physical layer specifications," ANSI/IEEE Std 802.4-1990 (Revision of ANSI/IEEE 802.4-1985), 1990.

[24] "IEEE Standard for Local and metropolitan area networks Media Access Control (MAC) Bridges," IEEE Std 802.1D-2004 (Revision of IEEE Std 802.1D-1998), 2004.

[25] "IEEE standards for local and metropolitan area networks: virtual bridged local area networks," IEEE Std 802.1Q, 1999.

[26] P. Neumann, "Communication in industrial automation-what is going on?," Control Engineering Practice, vol. 15, pp. 1332 - 47, 2007.

[27] E. Jasperneite and P. Neumann, "Switched Ethernet for factory communication," 8th International Conference on Emerging Technologies and Factory Automation (ETFA), vol. 1, pp. 205 - 212, 2001.

[28] S. Varadarajan and T.-C. Chiueh, "EtheReal: A host-transparent real-time fast Ethernet switch," International Conference on Network Protocols (ICNP), pp. 12 - 21, 1998.

[29] H. Hoang and M. Jonsson, "Switched real-time Ethernet in industrial applications: asymmetric deadline partitioning scheme," 2nd Internation Workshop on Real-Time LANs in the Internet Age, 2003.

[30] P. Pedreiras and L. Almeida, "Approaches to enforce real-time behavior in Ethernet," Industrial Communication Technology Handbook, R. Zurawski: CRC Press LLC, 2005, pp. 20-1-20-28.

[31] A. Takagi, S. Yamada, and S. Sugawara, "CSMA/CD with deterministic contention resolution," IEEE Journal on Selected Areas in Communications, vol. 1, pp. 877 - 884, 1983.

[32] G. Le Lann and N. Rivierre, "Real-Time Communications over Broadcast Networks: the CSMA-DCR and the DOD-CSMA-CD Protocols," INRIA 1863, 1993.

[33] C. L. Liu and J. W. Layland, "Scheduling Algorithms for multiprogramming in a hard environment," Journal ACM, vol. 20, pp. 46-61, 1973.

[34] P. M. Gopal and J. W. Wong, "Analysis of a hybrid token-CSMA/CD protocol for bus networks," Computer Networks and ISDN Systems, vol. 9, pp. 131 - 141, 1985.

[35] F. Carreiro, R. Moraes, J. A. Fonseca, and F. Vasques, "Real-Time Communication in Unconstrained Shared Ethernet Networks: The Virtual Token-Passing Approach," 10th IEEE International Conference on Emerging Technologies and Factory Automation (ETFA), vol. 1, pp. 425 - 432, 2005.

[36] J. L. Sobrinho and A. S. Krishnakumar, "EQuB - Ethernet Quality of Service using black bursts," Conference on Local Computer Networks, pp. 286 - 296, 1998.

[37] R. Yavatkar, P. Pai, and R. Finkel, "Reservation-based CSMA protocol for integrated manufacturing networks," IEEE Transactions on Systems, MAN and Cybernetics, vol. 24, pp. 1247 - 1258, 1994.

[38] M. L. Molle and L. Kleinrock, "Virtual Time CSMA: Why two clocks are better than one," IEEE Transactions on Communications, vol. 33, pp. 919 - 933, 1985.

[39] W. Zhao and K. Ramamritham, "Virtual time CSMA protocols for hard real-time communication," IEEE Transactions on Software Engineering, vol. SE-13, pp. 938 952, 1987. 
[40] R. Gallager, "Conflict resolution in random access broadcast networks," AFOSR Workshop Communication Theory Applications, pp. 74-76, 1978.

[41] J. F. Kurose, M. Schwartz, and Y. Yemini, "Multiple-access protocols and time-constrained communication," Computing Surveys, vol. 16, pp. 43 - 70, 1984.

[42] J. F. Kurose, M. Schwartz, and Y. Yemini, "Controlling window protocols for time-constrained communication in multiple access networks," IEEE Transactions on Communications, vol. 36, pp. 41 - 49, 1988.

[43] R. H. Jan and Y. J. Yeh, "CSMA/CD protocol for time-constrained communication on bus networks," IEE Proceedings, Part I: Communications, Speech and Vision, vol. 140, pp. 197 - 202, 1993.

[44] S. K. Kweon and K. G. Shin, "Achieving real-time communication over Ethernet with adaptive traffic smoothing," Proceedings Sixth IEEE Real-Time Technology and Applications Symposium, pp. 90 - 100, 2000.

[45] M. Butto, E. Cavallero, and A. Tonietti, "Effectiveness of the 'leaky bucket' policing mechanism in ATM networks," IEEE Journal on Selected Areas in Communications, vol. 9, pp. 335 - 42, 1991.

[46] J. Son, I.-G. Lee, H.-J. Yoo, and S.-C. Park, "An effective polling scheme for IEEE 802.11e," IEICE Transactions on Communications, vol. E88-B, pp. 4690 - 4693, 2005.

[47] D. Gao, J. Cai, and C. W. Chen, "Admission Control Based on Rate-Variance Envelop for VBR Traffic Over IEEE 802.11e HCCA WLANs," IEEE Transactions on Vehicular Technology, vol. 57, pp. 1778-1788, 2008.

[48] M. M. Rashid, E. Hossain, and V. K. Bhargava, "Controlled Channel Access Scheduling for Guaranteed QoS in 802.11e-Based WLANs," IEEE Transactions on Wireless Communications, vol. 7, pp. 1287-1297, 2008.

[49] S. C. Lo, G. Lee, and W. T. Chen, "An efficient multipolling mechanism for IEEE 802.11 wireless LANs," IEEE Transactions on Computers, vol. 52, pp. 764 - 68, 2003.

[50] D. Skyrianoglou, N. Passas, and A. K. Salkintzis, "ARROW: An Efficient Traffic Scheduling Algorithm for IEEE 802.11e HCCA," IEEE Transactions on Wireless Communications, vol. 5, pp. 3558-3567, 2006.

[51] S. Lee, K. N. Ha, J. H. Park, and K. C. Lee, "NDIS-based virtual polling algorithm of IEEE 802.11b for guaranteeing the real-time requirements," IECON Proceedings (Industrial Electronics Conference), pp. 2427 - 2432, 2005.

[52] M. Ergen, D. Lee, R. Sengupta, and P. Varaiya, "WTRP-wireless token ring protocol," IEEE Transactions on Vehicular Technology, vol. 53, pp. 1863 - 1881, 2004.

[53] R.-G. Cheng, C.-Y. Wang, L.-H. Liao, and J.-S. Yang, "Ripple: a wireless token-passing protocol for multi-hop wireless mesh networks," IEEE Communications Letters, vol. 10, pp. 123 - 5, 2006.

[54] A. Willig, "A MAC protocol and a scheduling approach as elements of a lower layers architecture in wireless industrial LANs," IEEE International Workshop on Factory Communication Systems (WFCS'97), pp. 139 - 48, 1997.

[55] F. De Pellegrini, D. Miorandi, S. Vitturi, and A. Zanella, "On the use of wireless 
networks at low level of factory automation systems," IEEE Transactions on Industrial Informatics, vol. 2, pp. 129 - 143, 2006.

[56] J. L. Sobrinho and A. S. Krishnakumar, "Quality-of-service in ad hoc carrier sense multiple access wireless networks," IEEE J. Sel. Areas Commun, vol. 17, pp. 1353 68, 1999.

[57] G.-H. Hwang and D.-H. Cho, "New access scheme for VoIP packets in IEEE 802.11e wireless LANs," IEEE Communications Letters, vol. 9, pp. 667 - 669, 2005.

[58] J.-P. Sheu, C.-H. Liu, S.-L. Wu, and Y.-C. Tseng, "A Priority MAC Protocol to Support Real-Time Traffic in Ad Hoc Networks," Wireless Networks, vol. 10, pp. 61 69, 2004.

[59] R. Moraes, F. Vasques, P. Portugal, and J. A. Fonseca, "VTP-CSMA: A Virtual Token Passing Approach for Real-Time Communication in IEEE 802.11 Wireless Networks," IEEE Transactions on Industrial Informatics, vol. 3, pp. 215-224, 2007.

[60] "SpectraLink Voice Priority - Quality of Service for voice traffic in wireless LANs," SpectraLink Wireless@Work.

[61] G.-H. Hwang and D.-H. Cho, "Fast retransmission mechanism for VoIP in IEEE 802.11e wireless LANs," IEEE 60th Vehicular Technology Conference (VTC2004-Fall), vol. 7, pp. 4996 - 5000, 2004.

[62] J. Deng and R. S. Chang, "A priority scheme for IEEE 802.11 DCF access method," IEICE Trans. Commun. (Japan), vol. E82-B, pp. 96 - 102, 1999.

[63] R. Moraes, P. Portugal, and F. Vasques, "Simulation analysis of the IEEE 802.11e EDCA protocol for an industrially-relevant real-time communication scenario," 11th IEEE International Conference on Emerging Technologies and Factory Automation (ETFA), pp. 202 - 209, 2006.

[64] N. J. Villallón, P. Cuenca, L. Orozco-Barbosa, and A. Garrido, "B-EDCA: A QoS mechanism for multimedia communications over heterogeneous 802.11/802.11e WLANs," Computer Communications, vol. 31, pp. 3905--3921, 2008.

[65] Y.-J. Wu, J.-H. Chiu, and T.-L. Sheu, "A modified EDCA with dynamic contention control for real-time traffic in multi-hop ad hoc networks," Journal of Information Science and Engineering, vol. 24, pp. 1065 - 1079, 2008.

[66] A. Hamidian and U. Korner, "An enhancement to the IEEE 802.11e EDCA providing QoS guarantees," Telecommunication Systems, vol. 31, pp. 195 - 212, 2006.

[67] "IEEE P802.11e/D10.0, Wireless LAN Medium Access Control (MAC) and Physical Layer (PHY) Specifications Amendment 7: Medium Access Control (MAC) Quality of Service Enhancements," 2005.

[68] C. Wang, B. Li, and L. Li, "A new collision resolution mechanism to enhance the performance of IEEE 802.11 DCF," IEEE Transactions on Vehicular Technology, vol. 53, pp. 1235 - 1246, 2004.

[69] X. Yang and N. Vaidya, "Priority scheduling in wireless ad hoc networks," Wireless Networks, vol. 12, pp. 273 - 286, 2006.

[70] N. Vaidya, A. Dugar, S. Gupta, and P. Bahl, "Distributed fair scheduling in a wireless LAN," IEEE Trans. Mob. Comput, vol. 4, pp. 616 - 29, 2005.

[71] S. J. Golestani, "A self-clocked fair queueing scheme for broadband applications," 
Proceedings IEEE INFOCOM '94. The Conference on Computer Communications. Networking for Global Communications, vol. 2, pp. 636 - 46, 1994.

[72] Y. Kwon, Y. Fang, and H. Latchman, "Design of MAC protocols with fast collision resolution for wireless local area networks," IEEE Transactions on Wireless Communications, vol. 3, pp. 793 - 807, 2004.

[73] E. Lopez-Aguilera, J. Casademont, J. Cotrina, and A. Rojas, "Enhancement proposal for WLAN IEEE 802.11e: Desynchronization of its Working Procedure," 14th IEEE Workshop on Local and Metropolitan Area Networks LANMAN, pp. 1-6, 2005.

[74] L. Lo Bello, F. S. Kaczynski, and O. Mirabella, "A Wireless traffic smoother for soft real-time communications over IEEE 802.11 industrial networks," 11th IEEE International Conference on Emerging Technologies and Factory Automation (ETFA), pp. 1073-1079, 2006.

[75] D. Miorandi and S. Vitturi, "Analysis of master-slave protocols for real-time industrial communications over IEEE 802.11 WLANs," 2nd IEEE International Conference on Industrial Informatics, pp. 143 - 148, 2004.

[76] G. Bianchi, I. Tinnirello, and L. Scalia, "Understanding 802.11e Contention-based Prioritization Mechanisms and their Coexistence with Legacy 802.11 Stations," IEEE Network, vol. 19, pp. 28 - 34, 2005.

[77] J. R. Moyne and D. M. Tilbury, "The Emergence of Industrial Control Networks for Manufacturing Control, Diagnostics, and Safety Data," Proceedings of the IEEE, vol. 95, pp. 29-47, 2007. 